\title{
Phenotypic analyses of rice Ise2 and Ise 3 mutants that exhibit hyperaccumulation of starch in the leaf blades
}

\author{
Chenggang Liang ${ }^{1,4 \dagger}$, Tatsuro Hirose ${ }^{1,2+}$, Masaki Okamura', Rei Tanimoto', Akio Miyao ${ }^{3}$, Hirohiko Hirochika ${ }^{3}$,
} Tomio Terao ${ }^{2}$, Tian $\mathrm{Li}^{4^{*}}$, Ryu Ohsugi ${ }^{1}$ and Naohiro Aoki ${ }^{*}$

\begin{abstract}
Background: To identify genes that potentially regulate the accumulation, mobilization, and transport of photoassimilates in rice (Oryza sativa L.) leaves, we recently screened a mutant collection of rice by iodine staining to visualize leaf starch contents. From this screening, we isolated a rice mutant that exhibits hyperaccumulation of starch in leaves and designated it as the Leaf Starch Excess 1 (LSE1) mutant. Here, we report two other rice LSE mutants, LSE2 and LSE3.

Results: Unlike Ise1 plants, Ise2 and Ise3 plants displayed retarded growth; Ise2 showed an extremely dwarf phenotype and rarely survived in paddy fields; Ise3 showed inhibited growth with pale green leaf blades, low tiller numbers, reduced height, and low grain yield. In Ise2 and Ise3 plants, the mature source leaves contained larger amounts of starch and sucrose than those in wild-type and Ise1 plants. Furthermore, microscopic observations of leaf transverse sections indicated that hyperaccumulation of starch in chloroplasts of mesophyll and bundle sheath cells occurred in Ise2 and Ise3 plants, while that in vascular cells was noticeable only in Ise3 leaves.

Conclusions: The distinct phenotypes of these three LSE mutants suggest that the LSE2 and LSE3 mutations occur because of disruption of novel genes that might be involved in the path of sucrose transport from mesophyll cells to phloem sieve elements in rice leaves, the mechanism for which has not yet been elucidated.
\end{abstract}

Keywords: Leaf blade; Oryza sativa; Phloem loading; Photoassimilate partitioning; Starch excess phenotype; Sucrose

\section{Background}

In plants, appropriate carbon partitioning within or between tissues is important for growth, development, and reproduction. Photosynthetically assimilated carbon is converted principally into carbohydrates such as cellulose, starch, sucrose, and hexoses. While cellulose forms the plant's structure, starch and soluble sugars often accumulate within tissues as non-structural carbohydrates (NSCs) or move from source to sink tissues as translocating sugars, of which sucrose is the most universal

\footnotetext{
* Correspondence: lit@sicau.edu.cn; aaokin@mail.ecc.u-tokyo.ac.jp ${ }^{\dagger}$ Equal contributors

${ }^{4}$ Key Laboratory of Crop Ecophysiology and Farming System in Southwest China, Ministry of P.C. China, College of Agronomy, Sichuan Agricultural University, Chengdu, Sichuan 611130, China

'Graduate School of Agricultural and Life Sciences, The University of Tokyo, Tokyo 113-8657, Japan

Full list of author information is available at the end of the article
}

among plant species (for recent reviews, see Stitt and Zeeman 2012; Ruan 2014). Thus, genetic modification of carbohydrate partitioning has long been proposed in crop breeding (Braun et al. 2014).

Leaf starch is the most widespread and abundant storage carbohydrate, and is synthesized during the day and hydrolyzed to soluble sugars at night for export from leaves to sites of growth (Perez et al. 1971; Lloyd et al. 2005; Smith et al. 2005; Smith 2012; Stitt and Zeeman 2012). Mutants lacking function in genes that are involved in these metabolic processes often display 'starch excess (sex)' phenotypes that accumulate excess quantities of starch in the leaves. Since these phenotypes can be easily screened by iodine staining of mature leaves, the genes responsible for sex mutations have been extensively studied to explore the molecular mechanisms of carbohydrate partitioning in leaves. By identifying and

\section{实}

(c) 2014 Liang et al.; licensee Springer. This is an Open Access article distributed under the terms of the Creative Commons Attribution License (http://creativecommons.org/licenses/by/4.0), which permits unrestricted use, distribution, and reproduction in any medium, provided the original work is properly credited. 
characterizing sex genes, the starch degradation pathway in leaves has been well established in Arabidopsis thaliana (L.) Heynh. (for recent reviews, see Zeeman et al. 2010; Stitt and Zeeman 2012). However, the starch degradation pathway may vary among plant species. For example, disproportionating enzyme 2 , which catalyzes the production of glucose, is located in the cytosol in $A$. thaliana (Chia et al. 2004) but functions in the chloroplasts in Solanum tuberosum L. (potato; Lloyd et al. 2004). The relevance of maltose transport from chloroplast to cytosol in potato remains unclear (Niittylä et al. 2004; Lloyd et al. 2005). Meanwhile, despite the fact that Oryza sativa L. (rice) is a model grass species and a major crop that feeds more than half of the global population, the molecular mechanism of starch degradation in the rice leaf has not been elucidated. We recently reported a sex mutant by an iodine staining-based screening of a rice mutant collection (Hirose et al. 2013). Seedlings of this mutant accumulated excess starch in the leaf blades, and the mutant was designated Leaf Starch Excess 1 (LSE1). The LSE1 mutation was determined to be caused by the disruption of a gene encoding $\alpha$-glucan, water dikinase, OsGWD1 (Os06g0498400; RAP_DB; http://rapdb.dna.affrc.go.jp/). Despite hyperaccumulation of starch in the leaf blade, the rice lse1 mutation appeared to have no significant effect on vegetative growth, in contrast to the sex1/gwd1 of $A$. thaliana (Caspar et al. 1991; Yu et al. 2001; Ritte et al. 2002) and the gwd1 of Lotus japonicus (Regel) K. Larsen (Vriet et al. 2010).

Starch-excess phenomena can also be caused by inhibition of photoassimilate export from leaves. For example, cold-girdling of the stem induces the starch-excess phenomenon by impairing photoassimilate transport (e.g., Krapp et al. 1993; Slewinski et al. 2009). In $A$. thaliana and Zea mays L. (maize), disruption of genes for a sucrose transporter (SUT) involved in apoplastic phloem loading was found to cause the LSE phenotype (Gottwald et al. 2000; Slewinski et al. 2009). In addition to knockout mutants of phloem-loading SUTs, maize mutants including sed1/sxd1 (Russin et al. 1996), $t d y 1$ (Braun et al. 2006), tyd2 (Baker and Braun 2008), and psc1 (Slewinski and Braun 2010), were reported to show the LSE phenotype accompanied by inhibition of photoassimilate export from leaves. Although rice is closely related to maize, the molecular mechanism for phloem loading of photoassimilates in rice leaves has not been elucidated. A rice SUT, OsSUT1, was reported to localize in the phloem (Matsukura et al. 2000; Scofield et al. 2007). However, antisense suppression of OsSUT1 did not induce clear symptoms of blocked phloem loading in source leaves (Ishimaru et al. 2001; Scofield et al. 2002). More recently, using an OsSUT1 knockout mutant with the anther culture technique, Eom et al. (2012) excluded the possibility that OsSUT1 plays a major role in phloem loading. Another rice SUT, OsSUT2, was reported to play an essential role in photoassimilate export from source leaf blades in rice, while this SUT localizes to the tonoplast membrane of leaf mesophyll cells (Eom et al. 2011). Thus, the path of photoassimilate transport from mesophyll tissue to phloem sieve elements in rice leaves remains unclear (for recent reviews, see Eom et al. 2012; Braun et al. 2014).

Here, we report two other rice LSE mutants, designated as LSE2 and LSE3. To investigate the roles of the target genes conferring the LSE2 and LSE3 mutations, putative homozygously mutated lines were established for LSE2 and LSE3; carbohydrate contents and plant growth in $l s e 2$ and lse3 were compared with lse 1 and wild-type (WT) plants. The lse 2 plants displayed severely dwarf phenotype, probably caused by the hyperaccumulation of starch and sucrose in leaf blades and sheaths. In contrast, lse3 plants displayed growth impairment that was intermediate between that of $l s e 1$ and $l s e 2$ plants, and exhibited hyperaccumulation of starch and elevated sugar levels only in the leaf blades. These distinct phenotypes, in combination with different patterns of starch accumulation in leaf tissue, suggest that the roles of LSE2 and LSE3 in leaf carbohydrate partitioning differ from that of $L S E 1$, and that LSE2 and LSE3 are more likely to be involved in sucrose export from leaves than in starch degradation.

\section{Results}

\section{Establishment of Ise2 and Ise 3 mutants of rice}

To compare inheritance of the LSE2 and LSE3 phenotype to that of LSE1, segregation analysis was conducted for each LSE mutant line using the $\mathrm{M}_{3}$ generation from stain-negative $M_{2}$ plants (Table 1). Similar to LSE1, the stain-positive phenotype in LSE2 was segregated at a ratio of 0.22 , suggesting that the phenotype was due to a recessive mutation of a single gene. The segregation ratio of the stain-positive phenotype in LSE3 was 0.16;

Table 1 Segregation ratios of LSE1, LSE2, and LSE3 mutant lines

\begin{tabular}{lcccc}
\hline LSE mutation $^{\mathbf{a}}$ & Number of stain-positive plants & Number of stain-negative plants & Segregation ratio of stain-positive plants & $\boldsymbol{P}_{\text {-Value }}^{\mathbf{b}}$ \\
\hline LSE1 & 25 & 84 & 0.23 & 0.69 \\
LSE2 & 36 & 129 & 0.22 & 0.71 \\
LSE3 & 10 & 68 & 0.13 & 0.01 \\
\hline
\end{tabular}

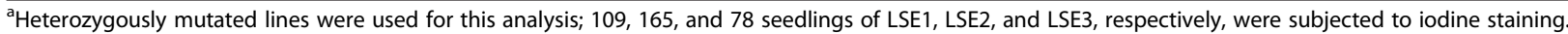

${ }^{b}$ Probability calculated by $\mathrm{X}^{2}$-test when the hypothetical segregation ratio of stain-positive plants is 0.25 . 
however, the LSE3 phenotype appeared to be caused by a recessive mutation of a single gene, because the ratio was significantly higher than the theoretical segregation ratio of 0.0625 that assumes two genes are responsible for the phenotype. This hypothesis was supported by the fact that a putative homozygously mutated line, in which all $\mathrm{M}_{3}$ individuals showed the stain-positive phenotype, was established for the LSE3 as well as for the LSE2 mutation (see Methods for details). Therefore, we decided to use the putative homozygous lines as the pure lines for the LSE2 and LSE3 mutations to characterize lse2 and lse3 plants, respectively. Meanwhile, similar to the LSE1 mutant line, Southern blot analysis revealed that neither the LSE2 nor the LSE3 phenotype was tagged by the Tos 17 retrotransposon, indicating that these phenotypes were caused by some other mutations (data not shown).

\section{Phenotypic comparisons of Ise1, Ise2, and Ise 3 plants grown under controlled glasshouse conditions}

To characterize lse 2 and lse 3 plants, we first compared them to lse1 and WT 'Nipponbare' plants at the seedling stage. Figure 1A shows representative results of iodine staining of lse1, lse2, lse3, and WT seedlings. The lse1 plants appeared to grow similarly to the WT, consistent with previous observations (Hirose et al. 2013). Compared to lse 1 and WT, both lse 2 and lse 3 exhibited impaired growth: lse 2 plants were very short and the height of lse 3 was intermediate between lse 1 and $l s e 2$. When seedlings were sampled just after the end of the 12-h dark period, almost no starch-accumulation signals were observed in any leaf blades of the WT. On the other hand, the fully matured leaf blades of lse seedlings were deeply stained with iodine. This result was consistent with TEM images of the transverse sections of leaves; many extraordinarily large starch granules were clearly observed in mesophyll chloroplasts of the three lse mutants (Figure $1 \mathrm{C}-\mathrm{E}$ ), while only a few small starch granules was observed in WT leaves (Figure 1B). To compare starch accumulation patterns in leaves, the transverse sections were stained with PAS solution to detect polysaccharides under light microscopy (Figure 2A-D). Again, deep staining was observed in all lse plants (Figure 2B-D) but not in WT plants (Figure 2A), showing starch hyperaccumulation or other polysaccharides in lse leaves. While deeply stained polysaccharides were observed not only in mesophyll cells, clear signals of polysaccharide accumulation were also detected in bundle sheath cells in leaves of the three lse mutants. The consistent presence of large starch granules in bundle sheath chloroplasts of lse leaves was observed under TEM, showing more prominent accumulation of starch compared to bundle sheath chloroplasts of WT leaves (Figure 2E-H). Furthermore, PAS-stained polysaccharides were often observed in the vascular tissues of lse3 leaves (Figure 2D), in vascular parenchyma cells (Kaneko et al. 1980; Chonan et al. 1981; Botha 2013). The presence of starch granules within the vascular tissue was evident only in lse3 (Figure $2 \mathrm{H}$ ).

Figure 3 shows 2-month-old WT, lse1, lse2, and lse3 plants grown in pots. As reported previously, the vegetative growth of lse1 was similar in appearance to the WT, with a slight decrease in tiller number (Hirose et al. 2013). Compared with WT and lse 1 plants, lse 2 displayed an extremely dwarf phenotype with yellowish leaves and no
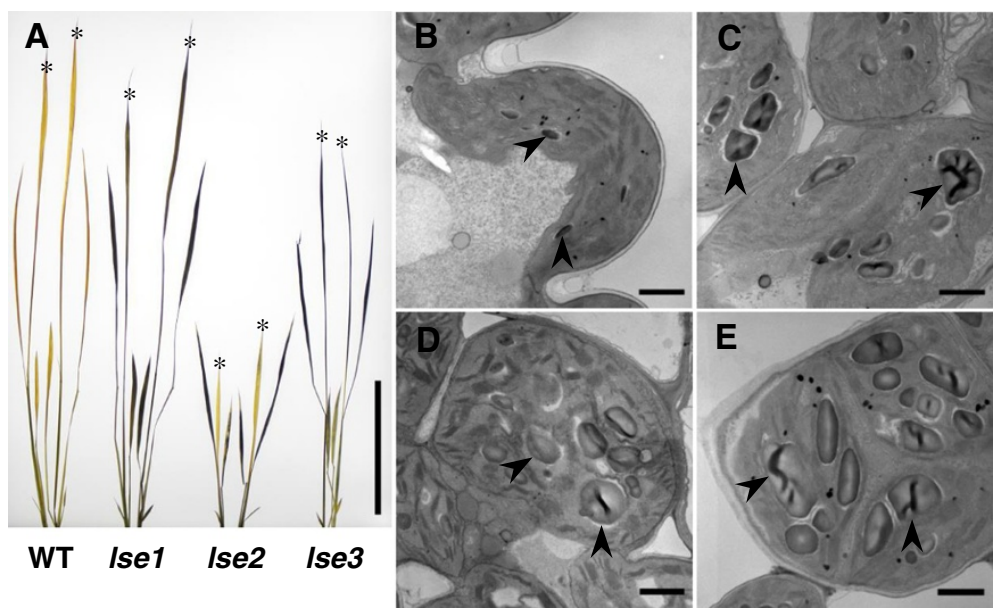

Figure 1 Starch-excess phenotypes of rice Ise mutants at the fifth leaf stage. Rice seedlings were sampled in the morning and subjected to iodine staining (A) or to microscopic observation (B-E). A, typical results of iodine staining in WT, Isel, Ise2, and Ise3 seedlings. Note that the youngest fifth leaf blades were still elongating very slowly and were not stained in 1 se2. Asterisks indicate the fifth leaf blade of rice seedlings. $\mathrm{Bar}=10 \mathrm{~cm}$. B-E, representative TEM images of transverse sections of fully-developed leaf blades of WT (B), Isel (C), Ise2 (D), and Ise3 (E). The fourth leaf blades were used for Ise2; fifth leaf blades were used for the others. Arrowheads indicate starch granules in mesophyll chloroplasts. Scale bars $=1 \mu \mathrm{m}$. 


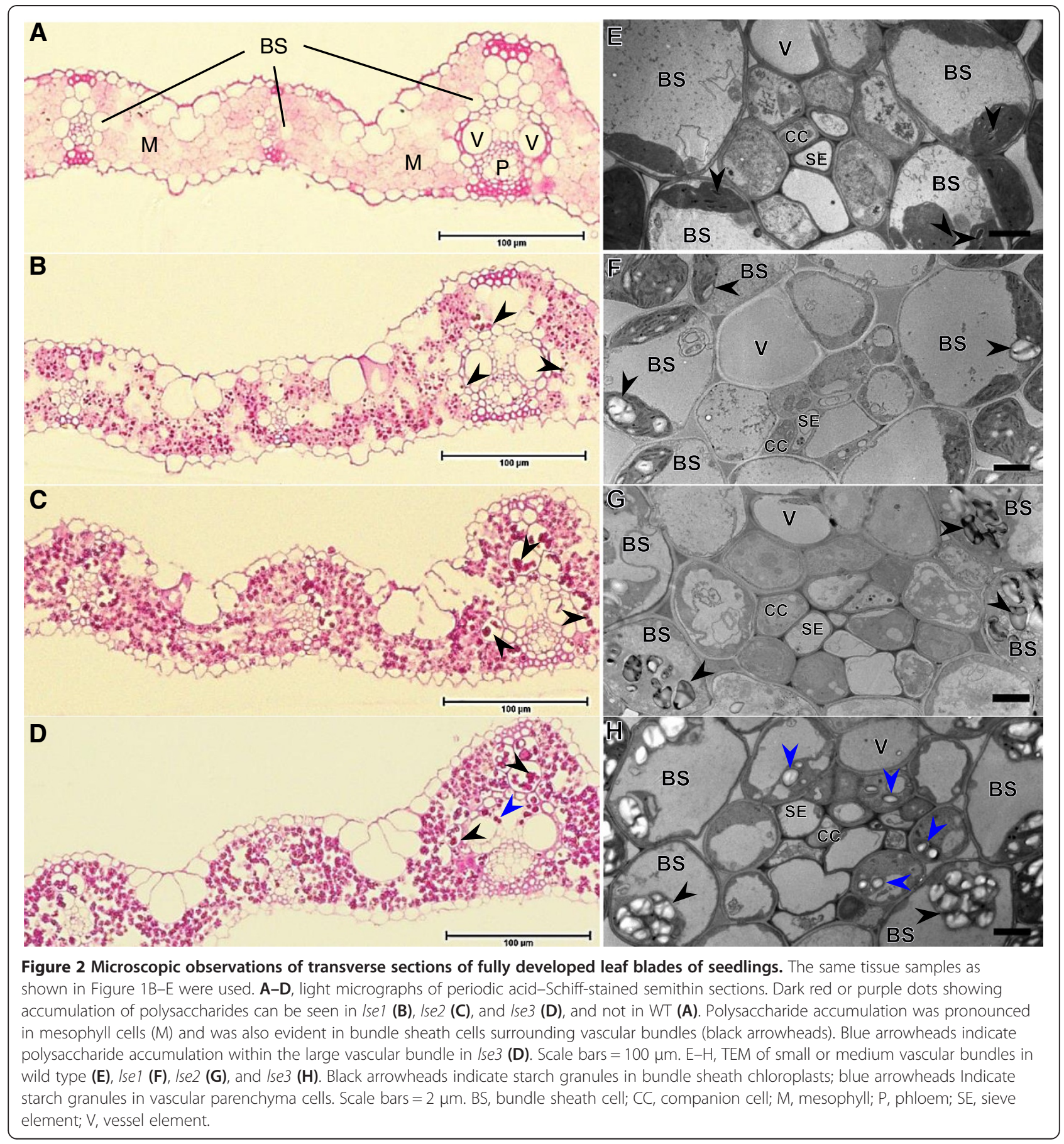

tiller, and rarely survived thereafter (Figure 3 ). The $l s e 3$ plants again exhibited a growth phenotype that was intermediate between that of $l s e 1$ and $l s e 2$ plants, with pale green leaves and no tiller.

To further characterize the accumulation profiles of NSCs in the lse plants, the concentrations of starch, sucrose, glucose, and fructose were determined in leaf blades and sheaths at the fifth-leaf stage (Figure 4), from samples collected at the beginning of the light (early morning) and the night (early evening) periods. Based on the iodine-staining patterns shown in Figure 1A, the leaf blades and sheaths of the fifth leaf of WT, lse1, lse2, and $l s e 3$ plants, and of the fourth leaf of $l s e 2$ plants were selected for analysis. In WT plants, as is common in source leaves of many plant species including rice, the NSC levels were lower in the morning and higher in the evening (Figure 4A,B; the data of statistical analysis are not shown). Levels of starch and soluble sugars were very 


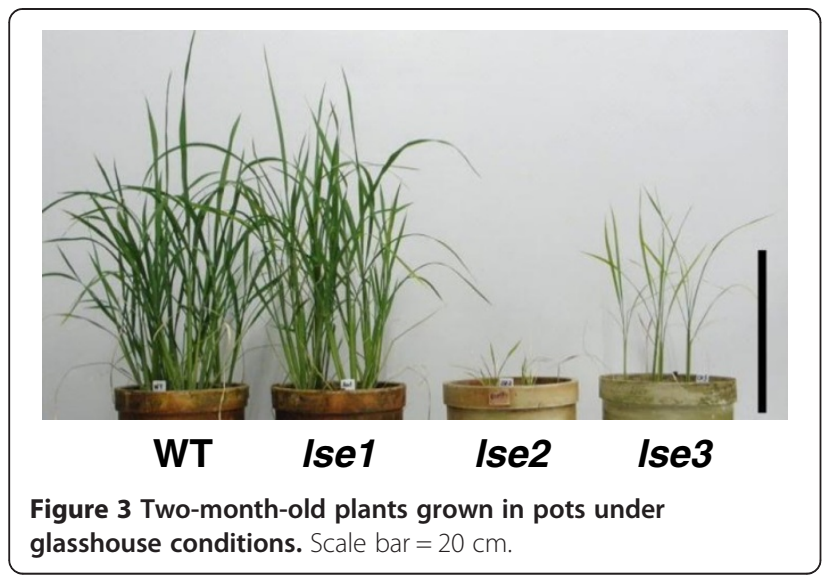

low in the morning because of nocturnal mobilization of NSCs stored in the leaves, and were high in the evening because of diurnal accumulation of excess photoassimilates in the form of NSCs. In comparison with WT leaves, none of the lse leaves showed clear day-night changes in NSC levels, suggesting that these leaves maintained extraordinarily high levels of NSCs (especially starch) throughout the day. Consistent with our previous findings (Hirose et al. 2013), starch levels in leaf blades of $l s e 1$ were significantly higher than that of WT in both morning and evening (Figure 4A,B), while soluble sugar levels in leaf blades did not differ statistically between lse1 and WT. As shown in Figure $1 \mathrm{~A}$, the fifth leaf blades of $l s e 2$ seedlings were still elongating and scarcely stained with iodine even when sampled in the early morning, while the fully-matured fourth leaf blades were deeply stained. This observation was quantitatively confirmed by the determination of NSC levels; no remarkable differences in starch level were observed between the fifth leaf blades of $l s e 2$ and WT, and starch levels in the fourth leaf blade of $l s e 2$ were much higher than those in the fifth leaf blade of WT, both in the morning and evening (Figure 4A,B). Soluble sugar (especially sucrose) levels in the mature leaf blades of $l s e 2$ were significantly higher than those of WT regardless of the sampling time. Starch and sugar levels in the leaf blades of lse3 were higher than those of the WT regardless of sampling time.

The leaf sheaths of grass species are considered to function as temporal storage tissues for accumulating excess photoassimilates, and as conducting tissues that connect source leaf blades with terminal sink tissues such as roots and developing leaves (for recent review see Slewinski 2012). In comparison with the WT, leaf sheaths of $l s e 1$ and les 3 contained significantly larger quantities of starch only in tissues sampled in the morning, and those of $l s e 2$ accumulated larger amounts of starch regardless of leaf age or sampling time (Figure 4C,D). Soluble sugar levels in leaf sheaths were similar between lse mutants and WT, or were lower in the mutants, except for the fourth

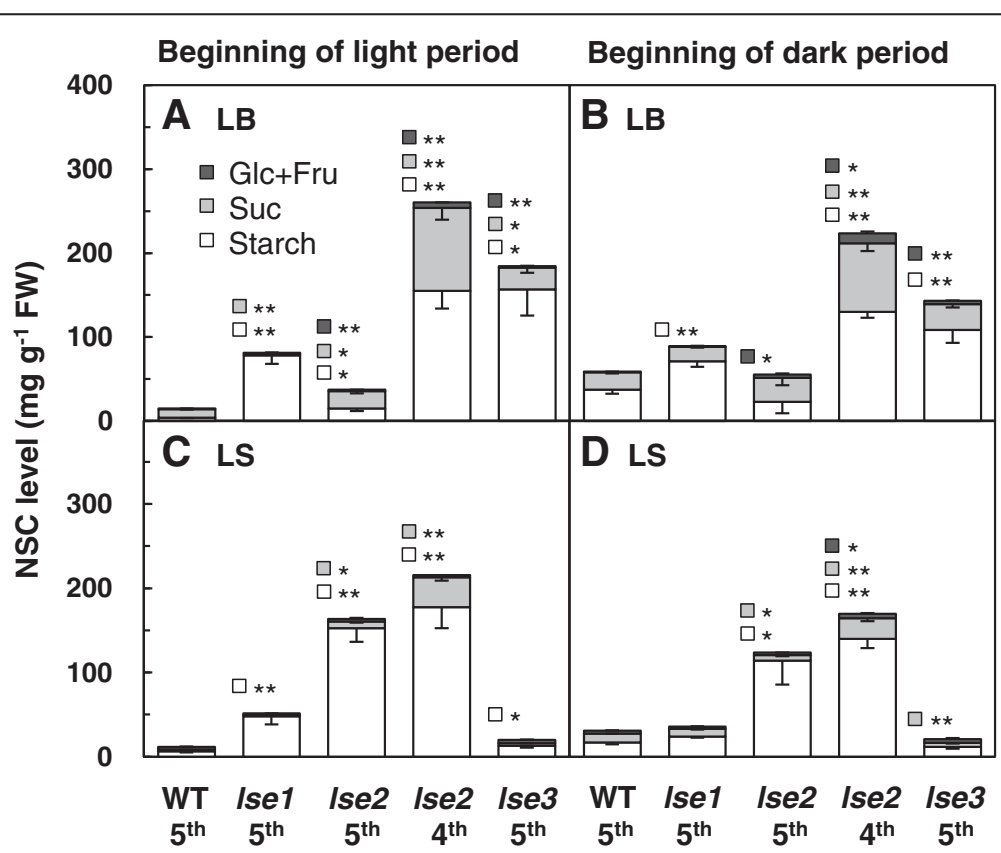

Figure 4 Comparison of concentrations of NSCs between WT and Ise mutants. Leaf blades (LB) and sheaths (LS) of fifth-leaf-stage seedlings were sampled at the beginning of the light period $(\mathbf{A}, \mathbf{C})$ and at the beginning of the dark period $(\mathbf{B}, \mathbf{D})$. The fifth leaf blades and sheaths $\left(5^{\text {th }}\right)$ were used for determination of starch, sucrose (Suc), glucose (Glc), and fructose (Fru). The fourth leaves $\left(4^{\text {th }}\right)$ were also used in Ise2. Values represent the averages of four plants \pm SE. Differences between the WT and each Ise mutant were analyzed by Student's $t$-test. Significant differences are indicated by asterisks ( $\left.{ }^{*} P \leq 0.05 ;{ }^{* *} P \leq 0.01\right)$. 
leaf sheath of $l s e 2$, in which sucrose levels were significantly higher than those of WT in both morning and evening.

\section{Phenotypic comparisons of Ise1, Ise2, and Ise3 plants grown under field conditions}

We also compared $l s e 2$ and $l s e 3$ plants to lse 1 and WT plants in terms of growth and productivity under field conditions, and obtained comparable results in two different years. Four-week-old seedlings were transplanted into a paddy field and grown in plant communities. The $l s e 2$ seedlings rarely survived under full-sun field conditions, requiring heavily shaded environments (e.g., surrounding one $l s e 2$ seedling with WT seedlings; data not shown) to persist. Therefore, we decided to use only lse3 for the phenotypic comparisons to lse1 and WT. First, we examined whether the phenotypic differences observed under glasshouse conditions were maintained in field-grown adult plants at the heading stage. Compared to lse 1 and WT plants, the heading day was delayed in lse3 plants by more than one week (data not shown), and the plant length of lse3 was significantly shorter (Table 2). These phenotypic characteristics were consistent with the retardation and impaired growth observed in $l s e 3$ (and $l s e 2$ ) at the seedling and vegetative growth stages (Figures $1 \mathrm{~A}$ and 3 ).

Next, NSC contents in leaf blades, sheaths, and internodes were compared at the heading stage (Figure 5). In leaf blades, starch levels were significantly higher in lse1 and lse 3 than in WT, showing the LSE phenotype in both the flag (top) and second-top leaf (Figure 5A,B). Starch levels in leaf sheaths and internodes were similar between lse1 and WT regardless of the position (Figure 5C-F). Compared to the WT, starch levels in lse3 plants tended to be lower in leaf sheaths and higher in internodes. Similar to the leaf blades of seedlings collected at the beginning of the light period (Figure 4B), soluble sugar (especially sucrose) levels were significantly elevated in leaf blades of $l s e 3$ plants compared with those of WT and lse1 plants (Figure 5A,B). In leaf sheaths and internodes, no significant increases in soluble sugar levels were observed in the two lse mutants compared with WT plants (Figure 5C-F). Finally, we measured the yield components at harvest (approximately 6 weeks after heading) (Table 2). As reported previously, the grain yield per plant was significantly decreased in lse1 compared with the WT because of a decrease in all four components: number of panicles per plant, number of spikelets per panicle, percentage of filled grains, and average grain weight (Hirose et al. 2013). The grain yield of $l s e 3$ was also approximately $70 \%$ lower than that of the WT because of considerable reductions in all the components except for percentage of filled grains (Table 2). It should be noted that the shaded $l s e 2$ plants were very short $(<50$ $\mathrm{cm}$ ) and showed little grain yield (approximately 5\% of WT) with no tillers, a small number of spikelets per panicle, a very low percentage of filled grains, and very small grain size.

\section{Discussion}

We previously reported screening of a rice mutant collection by iodine staining of seedlings harvested in the early morning (Hirose et al. 2013), and established a single-recessive rice mutant (lse1) that accumulates excess starch. In that paper, the responsible gene, LSE1, was identified as encoding an $\alpha$-glucan, water dikinase involved in starch degradation (OsGDW1; Os06g0498400; RAP_DB). Using similar procedures, we obtained two other rice LSE mutants, $l s e 2$ and $l s e 3$. Whereas hyperaccumulation of starch appeared to occur in chloroplasts in all three LSE mutants (Figure 1), lse 2 and $l s e 3$ showed phenotypes that were distinct from lse1. Unlike lse1 plants, lse 2 and lse 3 plants showed markedly impaired growth (Figures 1 and 3) and accumulated excess starch and sucrose in mature leaf blades (Figure 4). Segregation analysis suggested that the LSE2 phenotype was caused by a recessive mutation in a single gene, while the segregation ratio for the LSE3 phenotype implied non-Mendelian inheritance (Table 1). Although the reasons for the abnormal segregation ratio in LSE3 (0.16) remain to be clarified, it can be assumed that the phenotype is not a result of recessive mutations in two or more genes. Preliminary data from our ongoing gene-mapping work suggest that the LSE3 mutation is located in a single locus (data not shown). Collectively, either $l s e 2$ or $l s e 3$ would be a result of disruption of a single gene distinct from LSE1 (located on chromosome 6), although neither LSE2 nor LSE3 has been identified.

Table $\mathbf{2}$ Characteristics of rice plant growth and yield components

\begin{tabular}{|c|c|c|c|c|c|c|}
\hline & Plant length ${ }^{\mathrm{a}}(\mathrm{cm})$ & $\begin{array}{l}\text { Panicles } \\
\text { per plant }\end{array}$ & $\begin{array}{l}\text { Spikelets per } \\
\text { panicle }\end{array}$ & $\begin{array}{l}\text { Percentage of } \\
\text { filled grains (\%) }\end{array}$ & $\begin{array}{c}\text { Average grain } \\
\text { weight (mg) }\end{array}$ & Yield per plant (g) \\
\hline$\overline{W T}$ & $95.6 \pm 0.9$ & $10.8 \pm 0.3$ & $103.1 \pm 5.9$ & $76.5 \pm 2.4$ & $25.5 \pm 0.4$ & $22.0 \pm 1.9$ \\
\hline Isel & $92.2 \pm 1.1^{*}$ & $7.8 \pm 0.5^{* *}$ & $84.0 \pm 3.9^{*}$ & $70.2 \pm 2.7$ & $25.1 \pm 0.3$ & $11.4 \pm 0.5^{* *}$ \\
\hline Ise3 & $78.6 \pm 1.1^{* *}$ & $7.8 \pm 0.5^{* *}$ & $47.8 \pm 2.2^{* *}$ & $70.5 \pm 3.5$ & $24.2 \pm 0.2^{* *}$ & $6.4 \pm 0.7^{* *}$ \\
\hline
\end{tabular}

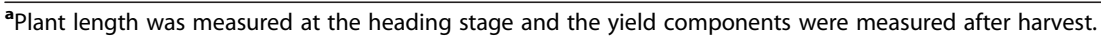

Values represent the means $\pm S E(n \geq 4)$. Differences between the WT and each /se mutant were analyzed by Student's t-test. Significant differences are indicated by asterisks $\left({ }^{*} P \leq 0.05 ;{ }^{* *} P \leq 0.01\right)$. 


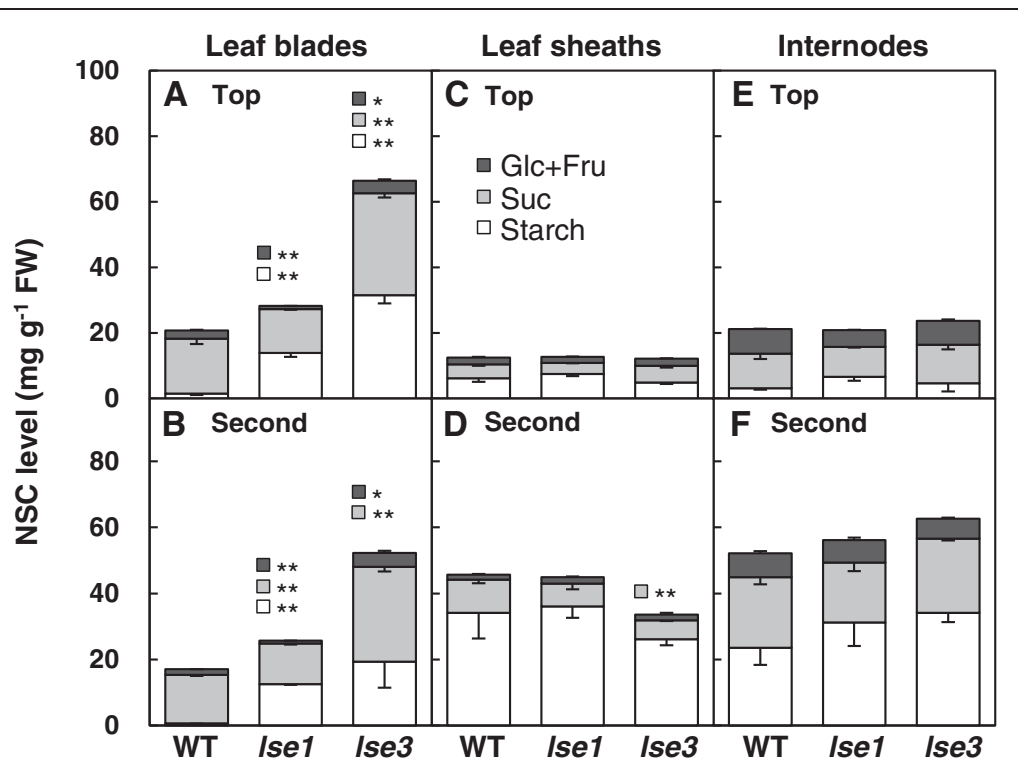

Figure 5 Comparison of concentrations of NSCs between WT and Ise mutants grown under field conditions. Leaf blades, sheaths, and internodes were sampled between 9:00 and 10:00 AM at the heading stage. The flag (top) leaf blades (A) and sheath (C), the second-top leaf blades (B) and sheaths (D), the top (E) and second-top (F) internodes were used for determination of starch, sucrose (Suc), glucose (Glc), and fructose (Fru). Values represent the averages of four plants \pm SE. Differences between the WT and each Ise mutant were analyzed by Student's $t$-test. Significant differences are indicated by asterisks $\left({ }^{*} P \leq 0.05 ;{ }^{* *} P \leq 0.01\right)$.

To date, single-gene disruption mutants that accumulate excess starch in source leaves have been reported in A. thaliana, L. japonicus, Solanum lycopersicum L. (tomato), maize, and rice (Additional file 1: Table S1, and references therein). Starch excess in leaves is often observed when inhibition of starch degradation occurs in mesophyll cells. No elevation in sugars, or a slight increase in sucrose and/or hyperaccumulation of maltose, has also been observed in these mutants (see Zeeman et al. 2010 for review). Any inhibition in export of photoassimilate from leaves can induce starch excess in the leaves (Additional file 1: Table S1, and references therein). In such LSE mutants in $A$. thaliana and maize, excess starch and sucrose tend to accumulate in leaves. Similarly, we found in rice $l s e 2$ and $l s e 3$ that both starch and sucrose levels in mature leaf blades of seedlings were significantly elevated in comparison with WT (Figure 4). We also found, in an HPLC analysis of carbohydrate contents, that none of $l s e 1, l s e 2, l s e 3$, or WT contained maltose at detectable levels in leaf blades (Additional file 2: Figure S1). This indicates that neither lse2 nor lse3 appear to hyperaccumulate maltose, which was found in a few LSE mutants of $A$. thaliana lacking genes (e.g., DPE2) for starch-degrading enzymes (Chia et al. 2004; Lu and Sharkey 2004) or for the chloroplast maltose transporter (e.g., MEX1; Niittylä et al. 2004). Thus, the characteristic carbohydrate profiles of rice $l s e 2$ and lse 3 suggest that photoassimilate export, rather than starch degradation, is inhibited. In fact, no elevation in sucrose levels was observed in the leaves of lse1 seedlings (Figure 4), which lack a starch-degrading enzyme, GWD1 (Hirose et al. 2013).

As described above, the phloem-localized OsSUT1 does not play an essential role in the phloem-loading mechanism in rice leaves, but the mesophyll (tonoplast)-localized OsSUT2 does play an important role. Since there are five members of the OsSUT gene family (Aoki et al. 2003), it is possible that the other three SUTs (OsSUT3, 4, and/or 5) are involved in apoplastic loading of sucrose in the phloem. However, we found, through an extensive expression analysis of OsSUT genes, that the transcripts for OsSUT3 and OsSUT5 were extremely low or undetectable in mature leaf blades of rice and that the transcript levels of OsSUT4 were much lower than those of OsSUT1 and did not differ between mesophyll tissues and vascular bundle (Additional file 3: Figure S2; also see Aoki et al. 2003). These results, together with the findings for OsSUT1 and OsSUT2, imply that rice phloem is not loaded by a SUT so a different loading mechanism (e.g., symplastic loading) may be working in source leaf blades. Since either the ossut1 or ossut 2 mutant has been reported not to exhibit LSE phenotype (Eom et al. 2011), we expect that LSE2 and LSE3 may be genes that differ from any OsSUTs and function in the yet-to-be-elucidated phloem loading mechanism in rice leaves.

In relation to the phloem loading mechanism in rice leaves, the concept of revised diffusion, proposed by Eom et al. (2012), is intriguing. This hypothesis is substantially identical to that suggested by Rennie and Turgeon (2009). In both reports, the authors hypothesized that passive 
diffusion of sucrose from the mesophyll to the phloem is accompanied by active pumping of sucrose from the vacuole into the cytosol of the mesophyll cells to maintain higher concentrations of sucrose in the cytosol. An important prerequisite for this hypothesis is that the symplastic connection between the phloem companion cell and the surrounding cells is adequate to allow diffusion of sucrose into the phloem. This requirement appears to be satisfied in rice, according to vein anatomy reported by Kaneko et al. (1980) and fluorescent dye movement observed by Scofield et al. (2007). Given that the symplastic pathway is the major route of phloem loading of sucrose in rice leaf blades, inhibition of sucrose export in the leaves of $l s e 2$ and lse 3 might be caused by loss of function in the plasmodesmata. In well-characterized maize sed1/ sxd1 mutant, export of photoassimilate from mature leaves appears to be inhibited by structural abnormalities in plasmodesmata connecting bundle sheath cells and vascular parenchyma cells, although the involvement of SED1/SXD1 (tocopherol cyclase) in plasmodesmata remains unclear (Russin et al. 1996; Botha et al. 2000; Provencher et al. 2001; Sattler et al. 2003). We have not observed structural abnormalities in plasmodesmata in leaf blades of $l s e 2$ or $l s e 3$ seedlings under TEM (data not shown). It would first be necessary to identify and characterize the responsible gene, including an in-situ expression analysis, prior to further investigation of the ultrastructure of cells around and within vascular bundles.

The severely impaired growth phenotypes of $l s e 2$ plants (Figures 1 and 3) indicate that the biochemical function of LSE2 is very likely to play a pivotal role in photoassimilate partitioning in rice plants. In transverse sections of $l s e 2$ leaves, hyperaccumulation of starch was observed in mesophyll and bundle sheath cells (Figure 2C,G), suggesting that inhibition may occur in the bundle sheath cells. It is possible that the photoassimilate (sucrose) cannot move into the inner cells of vascular bundles but that excess sucrose can be converted into starch within the bundle sheath cells. This hypothesis is supported by the fact that bundle sheath cells of rice leaf blades can synthesize starch from excess photoassimilate and can also probably degrade it, because hyperaccumulation of starch granules can be found in bundle sheath cells of $l s e 1$ leaves lacking a starch-degrading enzyme (Figure 2B,F). In addition to leaf blades, leaf sheaths of $l s e 2$ seedlings appear to accumulate large amounts of starch and sucrose (Figure 4C,D), suggesting a role for LSE2 in carbohydrate partitioning in leaf sheaths as well. LSE2 is likely to be involved in the remobilization of temporally stored NSCs, presumably functioning in the transport or metabolism of starch-derived sugars, although the apparent hyperaccumulation of NSCs in leaf sheaths may result from the very small volume of tissue in the dwarf plants (Figures 1 and 4). Meanwhile, it is intriguing that the starch-excess phenotype was evident in leaf sheaths of the fifth leaves of $l s e 2$ seedlings (Figure 4C,D) but not in the slowly developing leaf blade (Figure 1; Figure 4A,B). We cannot rule out the possibility that photoassimilates may be transported from the fourth (mature) leaves to the fifth (immature) leaf sheaths, leading to an excess of NSCs that triggers inhibition of photoassimilate partitioning and/or leaf tissue development in the fifth leaf blades.

In lse3 leaves, on the other hand, starch hyperaccumulation was observed not only in mesophyll and bundle sheath cells, but also within vascular tissues (Figure 2D,H), suggesting the role of LSE3 in photoassimilate transport for phloem loading; movement of sucrose from vascular parenchyma cells to the sieve element/companion cell complex may be inhibited in this mutant. This hypothesis could explain the elevated sucrose levels in leaf blades of lse3. However, the extent of inhibition of photoassimilate transport does not appear to be critical, as the impairment of growth in $l s e 3$ plants was not as severe as that observed in lse 2 plants (Figures 1 and 3 ) and the elevated leaf sugar levels in lse3 were not as remarkable as those in lse2. Interestingly, unlike leaf blades, leaf sheaths and internodes did not exhibit excess accumulation of starch or soluble sugars in lse3 (Figure 4C,D; Figure 5C-F), in contrast to lse 2 plants. In addition, there was no or only a slight reduction in the percentage of grain filling and grain weight in lse3 compared with lse1 and WT plants (Table 2), indicating that LSE3 is unlikely to play a major role in photoassimilate transport and partitioning in filling grains and sink tissues. These results suggest that the physiological importance of LSE3 is limited in the phloem loading pathway of photoassimilates in source leaves.

\section{Conclusions}

The results of phenotypic analysis suggest that LSE2 and LSE3 mutations are caused by the disruption of genes involved in the path of sucrose transport from mesophyll cells to phloem sieve elements in rice leaves. Meanwhile we cannot exclude the possibility that for either $l s e 2$ or lse3, the responsible gene is involved in other processes that can indirectly affect photoassimilate partitioning, such as cell and organelle development (Provencher et al. 2001) or sugar sensing and signaling (Ruan 2014). Further studies are necessary to characterize the biochemical and physiological functions of LSE2 and LSE3, by identifying LSE 2 and $L S E 3$ and by expression and localization analysis of these genes in different leaf tissues.

\section{Methods}

\section{Plant growth and sampling}

We previously reported screening of the Tos 17 retrotransposon-insertion mutant collection for rice cultivar 'Nipponbare' (Miyao et al. 2003; https://tos.nias.affrc.go.jp/) by iodine staining to detect starch accumulation in leaves of 
seedlings (stain-positive plants); as a result, five candidate lines were selected from more than 6300 lines of the $M_{2}$ generation (Hirose et al. 2013). In addition to a previously established LSE1 mutant line, we selected two other independent lines, which exhibited distinct starch accumulation in leaf blades of 4-week-old seedlings, in this study. During the original screening procedure using the $M_{2}$ generations, both the two lines segregated into stainpositive and stain-negative plants. To establish homozygously mutated lines, the progeny of stain-positive $\mathrm{M}_{2}$ plants were examined to check whether all individuals showed the stain-positive phenotype. On the basis of the results of this analysis, we selected the progeny of putative homozygous mutant plants to be the pure line for the mutation, designated the mutations as LSE2 and LSE3, and used these lines for further phenotypic analyses.

Seeds were grown in a plastic tray $(30 \times 3 \times 3 \mathrm{~cm})$ filled with nursery soil in a glasshouse (day/night cycle of $12 / 12 \mathrm{~h}, 25 / 20^{\circ} \mathrm{C} ; 60 \% \mathrm{RH}$ ) under natural light with supplementary lighting $\left(350 \mu \mathrm{mol} \mathrm{m} \mathrm{m}^{-2} \mathrm{~s}^{-1}\right)$. For starch staining, the aboveground parts of seedlings were boiled in $80 \%(\mathrm{v} / \mathrm{v})$ ethanol to remove pigments and stained with iodine solution. For the determination of NSCs, plants at the fifth-leaf stage were sampled at the end of the light or dark period, immediately frozen in liquid nitrogen, and stored at $-80^{\circ} \mathrm{C}$ until use.

The LSE mutant and WT ('Nipponbare') plants were also grown in paddy fields in 2012 and 2013, at the Institute for Sustainable Agro-ecosystem Services (ISAS), Tokyo, Japan $\left(35^{\circ} 44^{\prime} \mathrm{N}, 139^{\circ} 32^{\prime} \mathrm{E}\right)$. Seedlings were grown in a greenhouse for one month and transplanted into the paddy field in late May. The planting density was 22.2 hills $\mathrm{m}^{-2}$ (hill spacing of $30 \times 15 \mathrm{~cm}$ ) with one seedling per hill, and compound fertilizer for paddy fields $\left(\mathrm{N}: \mathrm{P}_{2} \mathrm{O}_{5}\right.$ : $\mathrm{K}_{2} \mathrm{O}=12: 16: 18 \%$ ) was applied at the rate of $50 \mathrm{~g} \mathrm{~m}^{-2}$ as a basal dressing. At the heading stage, following measurement of plant length, the upper tissues of WT, lse1, and lse 3 were sampled for measurement of NSCs, immediately frozen in dry ice, and stored at $-80^{\circ} \mathrm{C}$ until use. Plants were harvested in late September, approximately $45 \mathrm{~d}$ after heading, and their panicles were used for the analysis of yield components as described in Hirose et al. (2013).

\section{Determination of non-structural carbohydrates}

Frozen tissue samples harvested from seedlings were ground using a mortar and pestle under cryogenic conditions and transferred into a pre-weighted 2-mL microfuge tubes for measurement of fresh weight. For field-grown plants, frozen tissues were processed to a powder using a Multi-beads Shocker (MB901U, Yasui Kikai, Osaka, Japan) and transferred into pre-weighted 2-mL microfuge tubes for measurement of fresh weight. NSCs (i.e., starch, sucrose, glucose, and fructose) were extracted and measured as described previously (Hirose et al. 2013).

\section{Microscopic observations}

Fully expanded leaf blades of 4-week-old seedlings were collected in the morning. For each line, three seedlings were selected and the middle parts of the leaf blades were cut into small segments and used immediately for microscopic observation. The extent of starch accumulation was determined by iodine staining of the rest (tips and bottom parts) of the sampled leaves. The small segments of leaf blades were fixed in $4 \%(\mathrm{w} / \mathrm{v})$ paraformaldehyde and $2 \%(\mathrm{w} / \mathrm{v})$ glutaraldehyde in $50 \mathrm{mM}$ phosphate buffer ( $\mathrm{pH} 7.2)$ for $24 \mathrm{~h}$ and post-fixed with $2 \%(\mathrm{w} / \mathrm{v})$ osmium tetroxide aqueous solution in the same buffer for $2 \mathrm{~h}$ at $4^{\circ} \mathrm{C}$. Dehydration and resin infiltration were performed in a graded series of acetone solution and propylene oxide. The samples were embedded in Spurr's epoxy resin (Sigma-Aldrich, St. Louis, MO, USA). Ultrathin transverse sections were prepared with an ultramicrotome (Ultracut UTC, Leica, Germany) and stained with uranyl acetate and lead citrate, and observed using a transmission electron microscope (JEM-1010, JEOL Ltd., Tokyo, Japan) operated at $100 \mathrm{kV}$. Semithin transverse sections (1 $\mu \mathrm{m}$ thickness) were subjected to periodic acidSchiff (PAS) staining and observed using a light microscope (Eclipse Ti-S, Nikon, Tokyo, Japan).

\section{Additional files}

Additional file 1: Table S1. Leaf Starch Excess (LSE) mutants in angiosperms.

Additional file 2: Figure S1. Elution profiles of high performance anion-exchange chromatography (HPAEC) for analysis of soluble sugar contents in leaf blades.

Additional file 3: Figure S2. Real-time quantitative reverse-transcription PCR analysis of OSSUT genes in leaf blades of rice.

\section{Abbreviations}

Glc: Glucose; Fru: Fructose; LSE: Leaf starch excess; NSC: Non-structural carbohydrate; PAS: Periodic acid-Schiff; Suc: Sucrose; SUT: Sucrose transporter; WT: Wild type.

\section{Competing interests}

The authors declare that they have no competing interests.

\section{Authors' contributions}

$\mathrm{TH}, \mathrm{T}, \mathrm{RO}, \mathrm{TL}$ and $\mathrm{NA}$ designed the research project. $\mathrm{CL}, \mathrm{TH}$ and NA designed and performed all of the experiments, analyzed the data, and drafted the manuscript. MO and RT assisted the field experiments and the analysis of the data. AM and $\mathrm{HH}$ produced and provided the rice mutant collection. All authors read and approved the final manuscript.

\section{Acknowledgements}

The authors thank Dr. Fumiko Ishizuna of the Electron Microscope Laboratory, The University of Tokyo, for excellent assistance with microscopy; Kiiko Takatsuto, NARO Agricultural Research Center, for excellent technical support; the technical support staffs of the ISAS, The University of Tokyo, for cultivation management of rice; and the China Scholarship Council for the State Scholarship Fund (grant number 201206910010) to CL. This study was supported in part by the Japan Society for the Promotion of Science (JSPS) KAKENHI (grant number 22380017) to $\mathrm{TH}, \mathrm{NA}$, and RO. MO is a recipient of a fellowship from the JSPS. 


\section{Author details}

${ }^{1}$ Graduate School of Agricultural and Life Sciences, The University of Tokyo, Tokyo 113-8657, Japan. ${ }^{2}$ NARO Agricultural Research Center, Joetsu, Niigata 943-0193, Japan. ${ }^{3}$ National Institute of Agrobiological Sciences, Tsukuba, Ibaraki 305-8602, Japan. ${ }^{4}$ Key Laboratory of Crop Ecophysiology and Farming System in Southwest China, Ministry of P.C. China, College of Agronomy, Sichuan Agricultural University, Chengdu, Sichuan 611130, China.

Received: 24 July 2014 Accepted: 24 November 2014

Published online: 21 December 2014

\section{References}

Aoki N, Hirose T, Scofield GN, Whitfeld PR, Furbank RT (2003) The sucrose transporter gene family in rice. Plant Cell Physiol 44:223-232. doi:10.1093/pcp/pcg030

Baker RF, Braun DM (2008) Tie-dyed2 functions with Tie-dyed1 to promote carbohydrate export from maize leaves. Plant Physiol 146:1085-1097. doi:10.1104/pp. 104.900251

Botha CEJ (2013) A tale of two neglected systems - structure and function of the thin- and thick-walled sieve tubes in monocotyledonous leaves. Front Plant Sci 4:297. doi:10.3389/fpls.2013.00297

Botha CEJ, Cross RHM, van Bel AJE, Peter Cl (2000) Phloem loading in the sucrose-export-defective (SXD-1) mutant maize is limited by callose deposition at plasmodesmata in bundle sheath-vascular parenchyma interface. Protoplasma 214:65-72. doi:10.1007/BF02524263

Braun DM, Ma Y, Inada N, Muszynski MG, Baker RF (2006) Tie-dyed1 regulates carbohydrate accumulation in maize leaves. Plant Physiol 142:1511-1522. doi:10.1104/pp. 106.090381

Braun DM, Wang L, Ruan YL (2014) Understanding and manipulating sucrose phloem loading, unloading, metabolism, and signalling to enhance crop yield and food security. J Exp Bot 65:1713-1735. doi:10.1093/jxb/ert416

Caspar T, Lin TP, Kakefuda G, Benbow L, Preiss J, Somerville C (1991) Mutants of Arabidopsis with altered regulation of starch degradation. Plant Physiol 95:1181-1188. doi:10.1104/pp. 95.4.1181

Chia T, Thorneycroft D, Chapple A, Messerli G, Chen J, Zeeman SC, Smith SM, Smith AM (2004) A cytosolic glucosyltransferase is required for conversion of starch to sucrose in Arabidopsis leaves at night. Plant J 37:853-863. doi:10.1111/j.1365-313X.2003.02012x

Chonan N, Kaneko M, Kawahara H, Matsuda T (1981) Ultrastructure of the large vascular bundles in the leaves of rice plant. Jpn J Crop Sci 50:323-331

Eom J, Cho J, Reinders A, Lee S, Yoo Y, Tuan PQ, Choi S, Bang G, Park Y, Cho M (2011) Impaired function of the tonoplast-localized sucrose transporter in rice, OSSUT2, limits the transport of vacuolar reserve sucrose and affects plant growth. Plant Physiol 157:109-119. doi:10.1104/ pp. 111.176982

Eom J, Choi S, Ward JM, Jeon J (2012) The mechanism of phloem loading in rice (Oryza sativa). Mol Cells 33:431-438. doi:10.1007/s10059-012-0071-9

Gottwald JR, Krysan PJ, Young JC, Evert RF, Sussman MR (2000) Genetic evidence for the in planta role of phloem-specific plasma membrane sucrose transporters. Proc Natl Acad Sci U S A 97:13979-13984. doi:10.1073/pnas.250473797

Hirose T, Aoki N, Harada Y, Okamura M, Hashida Y, Ohsugi R, Miyao A, Hirochika H, Terao T (2013) Disruption of a rice gene for a-glucan water dikinase, OsGWD1, leads to hyperaccumulation of starch in leaves but exhibits limited effects on growth. Front Plant Sci 4:147. doi:10.3389/fpls.2013.00147

Ishimaru K, Hirose T, Aoki N, Takahashi S, Ono K, Yamamoto S, Wu J, Saji S, Baba T, Ugaki M (2001) Antisense expression of a rice sucrose transporter OsSUT1 in rice (Oryza sativa L.). Plant Cell Physiol 42:1181-1185. doi:10.1093/pcp/pce148

Kaneko M, Chonan N, Matsuda T, Kawahara H (1980) Ultrastructure of the small vascular bundles and transfer pathways for photosynthate in the leaves of rice plant. Jpn J Crop Sci 49:42-50. doi:10.1626/jcs.49.42

Krapp A, Hofmann B, Schäfer C, Stitt M (1993) Regulation of the expression of rbcS and other photosynthetic genes by carbohydrates: a mechanism for the 'sink regulation' of photosynthesis? Plant J 3:817-828. doi:10.1111/ j.1365-313X.1993.00817.x

Lloyd JR, Blennow A, Burhenne K, Kossmann J (2004) Repression of a novel isoform of disproportionating enzyme (stDPE2) in potato leads to inhibition of starch degradation in leaves but not tubers stored at low temperature. Plant Physiol 134:1347-1354. doi:10.1104/pp. 103.038026

Lloyd JR, Kossmann J, Ritte G (2005) Leaf starch degradation comes out of the shadows. Trends Plant Sci 10:130-137. doi:10.1016/ j.tplants.2005.01.001

Lu Y, Sharkey TD (2004) The role of amylomaltase in maltose metabolism in the cytosol of photosynthetic cells. Planta 218:466-473. doi:10.1007/ s00425-003-1127-z

Matsukura C, Saitoh T, Hirose T, Ohsugi R, Perata P, Yamaguchi J (2000) Sugar uptake and transport in rice embryo. Expression of companion cell-specific sucrose transporter (OsSUT1) induced by sugar and light. Plant Physiol 124:85-94. doi:10.1104/pp. 124.1.85

Miyao A, Tanaka K, Murata K, Sawaki H, Takeda S, Abe K, Shinozuka Y, Onosato K, Hirochika H (2003) Target site specificity of the Tos 17 retrotransposon shows a preference for insertion within genes and against insertion in retrotransposon-rich regions of the genome. Plant Cell 15:1771-1780 doi:10.1105/tpc.012559

Niittylä T, Messerli G, Trevisan M, Chen J, Smith AM, Zeeman SC (2004) A previously unknown maltose transporter essential for starch degradation in leaves. Science 303:87-89. doi:10.1126/science.1091811

Perez CM, Palmiano EP, Baun LC, Juliano BO (1971) Starch metabolism in the leaf sheaths and culm of rice. Plant Physiol 47:404-408. doi:10.1104/ pp.47.3.404

Provencher LM, Miao L, Sinha N, Lucas WJ (2001) Sucrose Export Defective1 encodes a novel protein implicated in chloroplast-to-nucleus signaling. Plant Cell 13:1127-1141. doi:10.1105/tpc.13.5.1127

Rennie EA, Turgeon R (2009) A comprehensive picture of phloem loading strategies. Proc Natl Acad Sci U S A 106:14162-14167. doi:10.1073/ pnas.0902279106

Ritte G, Lloyd JR, Eckermann N, Rottmann A, Kossmann J, Steup M (2002) The starch-related R1 protein is an a-glucan, water dikinase. Proc Natl Acad Sci U S A 99:7166-7171. doi:10.1073/pnas.062053099

Ruan YL (2014) Sucrose metabolism: Gateway to diverse carbon use and sugar signaling. Annu Rev Plant Biol 65:33-67. doi:10.1146/annurev-arplant050213-040251

Russin WA, Evert RF, Vanderveer PJ, Sharkey TD, Briggs SP (1996) Modification of a specific class of plasmodesmata and loss of sucrose export ability in the sucrose export defective1 maize mutant. Plant Cell 8:645-658. doi:10.1105/ tpc.8.4.645

Sattler SE, Cahoon EB, Coughlan SJ, DellaPenna D (2003) Characterization of tocopherol cyclases from higher plants and cyanobacteria. Evolutionary implications for tocopherol synthesis and function. Plant Physiol 132:2184-2195. doi:10.1104/pp. 103.024257

Scofield GN, Hirose T, Gaudron JA, Upadhyaya NM, Ohsugi R, Furbank RT (2002) Antisense suppression of the rice transporter gene, OsSUT1, leads to impaired grain filling and germination but does not affect photosynthesis. Funct Plant Biol 29:815-826. doi:10.1071/PP01204

Scofield GN, Hirose T, Aoki N, Furbank RT (2007) Involvement of the sucrose transporter, OSSUT1, in the long-distance pathway for assimilate transport in rice. J Exp Bot 58:3155-3169. doi:10.1093/jxb/erm153

Slewinski TL (2012) Non-structural carbohydrate partitioning in grass stems: a target to increase yield stability, stress tolerance, and biofuel production. J Exp Bot 63:4647-4670. doi:10.1093/jxb/ers124

Slewinski TL, Braun DM (2010) The Psychedelic genes of maize redundantly promote carbohydrate export from leaves. Genetics 185:221-231. doi:10.1534/genetics.109.113357

Slewinski TL, Meeley R, Braun DM (2009) Sucrose transporterl functions in phloem loading in maize leaves. J Exp Bot 60:881-892. doi:10.1093/ jxb/ern335

Smith AM (2012) Starch in the Arabidopsis plant. Starch-Starke 64:421-434. doi:10.1002/star.201100163

Smith AM, Zeeman SC, Smith SM (2005) Starch degradation. Annu Rev Plant Biol 56:73-98. doi:10.1146/annurev.arplant.56.032604.144257

Stitt M, Zeeman SC (2012) Starch turnover: pathways, regulation and role in growth. Cur. Opin Biotechnol 3:282-292. doi:10.1016/j.pbi.2012.03.016

Vriet C, Welham T, Brachmann A, Pike M, Pike J, Perry J, Parniske M, Sato S, Tabata S, Smith AM (2010) A suite of Lotus japonicus starch mutants reveals both conserved and novel features of starch metabolism. Plant Physiol 154:643-655. doi:10.1104/pp. 110.161844

Yu TS, Kofler H, Häusler RE, Hille D, Flügge UI, Zeeman SC, Smith AM, Kossmann J, Lloyd J, Ritte G (2001) The Arabidopsis sex1 mutant is defective in the R1 
protein, a general regulator of starch degradation in plants, and not in the chloroplast hexose transporter. Plant Cell 13:1907-1918. doi:10.1105/ TPC.010091

Zeeman SC, Kossmann J, Smith AM (2010) Starch: its metabolism, evolution, and biotechnological modification in plants. Annu Rev Plant Biol 61:209-234. doi:10.1146/annurev-arplant-042809-112301

doi:10.1186/s12284-014-0032-3

Cite this article as: Liang et al:: Phenotypic analyses of rice Ise 2 and Ise 3 mutants that exhibit hyperaccumulation of starch in the leaf blades.

Rice 2014 7:32.

Submit your manuscript to a SpringerOpen ${ }^{\circ}$ journal and benefit from:

- Convenient online submission

- Rigorous peer review

- Immediate publication on acceptance

- Open access: articles freely available online

- High visibility within the field

- Retaining the copyright to your article

Submit your next manuscript at $>$ springeropen.com 